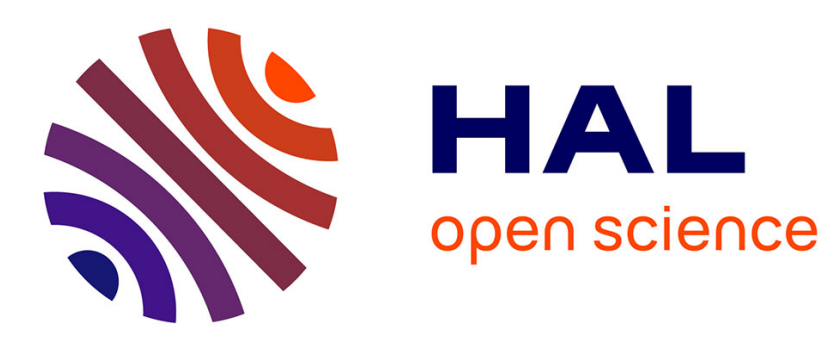

\title{
Optically Induced Coupling of Two Magnetic Dopant Spins by a Photoexcited Hole in a Mn-DopedInAs/GaAs Quantum Dot
}

\author{
Olivier Krebs, A Lemaître
}

\section{- To cite this version:}

Olivier Krebs, A Lemaître. Optically Induced Coupling of Two Magnetic Dopant Spins by a Photoexcited Hole in a Mn-DopedInAs/GaAs Quantum Dot. Physical Review Letters, 2013, 111, 10.1103/physrevlett.111.187401 . hal-03431818

\section{HAL Id: hal-03431818 \\ https://hal.science/hal-03431818}

Submitted on 16 Nov 2021

HAL is a multi-disciplinary open access archive for the deposit and dissemination of scientific research documents, whether they are published or not. The documents may come from teaching and research institutions in France or abroad, or from public or private research centers.
L'archive ouverte pluridisciplinaire HAL, est destinée au dépôt et à la diffusion de documents scientifiques de niveau recherche, publiés ou non, émanant des établissements d'enseignement et de recherche français ou étrangers, des laboratoires publics ou privés. 


\title{
Optically induced coupling of two magnetic dopant spins by a photoexcited hole in a Mn-doped InAs/GaAs quantum dot
}

\author{
O. Krebs and A. Lemaître \\ CNRS-Laboratoire de Photonique et de Nanostructures, Route de Nozay, 91460 Marcoussis, France
}

(Dated: October 10, 2013)

\begin{abstract}
We report evidence of a photo-induced coupling between two spins provided by Mn dopants in their neutral acceptor state $A^{0}$ in a single InAs/GaAs quantum dot. The coupling occurs due to simultaneous exchange interactions between each of the two dopant spins and a photo-created hole. Micro-photoluminescence spectroscopy achieved both in longitudinal and perpendicular magnetic field reveals the splitting of the 4 spin configurations $\left|J_{1}= \pm 1, J_{2}= \pm 1\right\rangle$ due to the $2 A^{0}$-hole exchange interaction. We obtain a comprehensive interpretation of the experimental data with a simplified spin Hamiltonian model, which more specifically shows that the hole-mediated coupling is similar to a $\varepsilon_{12} \simeq-70 \mu \mathrm{eV}$ exchange interaction between both $A^{0}$ spins.
\end{abstract}

PACS numbers: 78.67.Hc,75.50.Pp,71.70.Gm,75.10.Dg

Semiconductor quantum dots (QDs) have been extensively studied in the last decade to address and manipulate optically the spin of a single trapped electron or hole [1-7]. The controllable coupling of two such spins, aimed to perform quantum operations, is more challenging as it requires to deal with the indiscernibility of identical carriers. A solution consists in isolating both carriers in two distinct QDs which can be tunnel-coupled by a tunable electric field [8-11]. Alternatively, an individual QD containing two point-like spins, each provided by a single atom and with negligible mutual interaction, could be used to control their coupling through their respective interaction with a common electron or hole. This could be achieved with magnetic dopants indirectly coupled via the $s p$ - $d$ exchange interaction. Such magnetically doped QDs would be of particular interest because the strength of the exchange, as reported for singly Mndoped CdTe/ZnTe [12] or InAs/GaAs [13] QDs, is high enough to spectrally resolve the different magnetic spin states, and therefore to achieve their optical manipulation and readout, notably with few-picosecond pulses.

The optical spectroscopy of $\mathrm{CdTe} / \mathrm{ZnTe}$ QDs doped with two Mn atoms has been recently reported [14]. It revealed clear spin correlations induced by their overlapping with a common resident or photo-created carrier in the quantum dot and the possibility for optical control. Here, we present an analogous investigation of an InAs/GaAs QD containing two Mn atoms, relying on the magneto-optical spectroscopy in a longitudinal or perpendicular magnetic field and its interpretation within a simplified spin Hamiltonian model. Our results show that the magnetic dopants experience an effective exchange interaction induced by a photo-created hole. Besides many conceptual similarities with the II-VI system, the specific properties of Mn-doped III-V QDs [15, 16] make the interpretation somewhat simpler with essentially 4 spin configurations (against 36), more suitable for the design of a spin-based CNOT gate.

In the III-V $\operatorname{In}_{x} \mathrm{Ga}_{1-x} \mathrm{As}$ compound the magnetic dopant provided by a substitutional $\mathrm{Mn}$ atom forms an acceptor state denoted $A^{0}$ consisting of a negative center $A^{-}$on the Mn site and a bound hole in the host valence band with $\sim 1 \mathrm{~nm}$ Bohr radius [17]. The direct exchange interaction between two such acceptors must typically extend over twice their radius, in agreement with the direct imaging of $2 A^{0}$ wave-functions in GaAs subsurface [18]. In an InAs/GaAs QD of about $20 \mathrm{~nm}$ diameter and $4 \mathrm{~nm}$ height, the direct coupling between two randomly distributed $A^{0}$ should vanish in more than $95 \%$ of the cases. The effective spin of a magnetic acceptor $A^{0}$ is associated to its total angular momentum $J=1$ corresponding to the anti-ferromagnetic configuration of the Mn $3 d^{5}$ electrons ( $\operatorname{spin} S=5 / 2$ ) and the hole angular momentum $J_{\mathrm{h}}=3 / 2$ [19-21]. In a self-assembled InAs/GaAs QD presenting both lens shape and biaxial strain, the state $\left|J_{z^{\prime}}=0\right\rangle$ of the $J=1$ triplet, where $z^{\prime}$ is a Mn-site-dependent direction close to the growth axis $z$, is substantially shifted at higher energy (counting positively the hole energy) with respect to the states $\left|J_{z^{\prime}}= \pm 1\right\rangle$ by a few meV $[15,22]$. Consequently, this level is negligibly occupied at low temperature $(\lesssim 2 \mathrm{~K})$ and one generally observes the $A^{0}$ acceptor mostly in one of the two states $\left|J_{z^{\prime}}= \pm 1\right\rangle$.

We used the same Mn-doped photo-diode device as in Ref.'s 13 and 16 which consists of a QD layer embedded in between an electron reservoir and a semi-transparent Schottky gate enabling us to control the charge. The setup for micro-photoluminescence ( $\mu-P L)$ spectroscopy in magnetic field is described in the same references. PL spectra presented in this Letter were acquired at low temperature $(T \approx 1.7 \mathrm{~K})$ under non-resonant excitation (HeNe $633 \mathrm{~nm}$ laser line) in a magnetic field applied in the Faraday or Voigt configuration, i.e. parallel or perpendicular to the $z$ optical axis. In both cases, the PL signal was measured in $\sigma^{+}$circular polarization corresponding to the detection of a $|\Uparrow\rangle$-spin hole recombining with a $|\downarrow\rangle$-spin electron. The sample was studied in a regime where the QDs are charged by an electron, so 

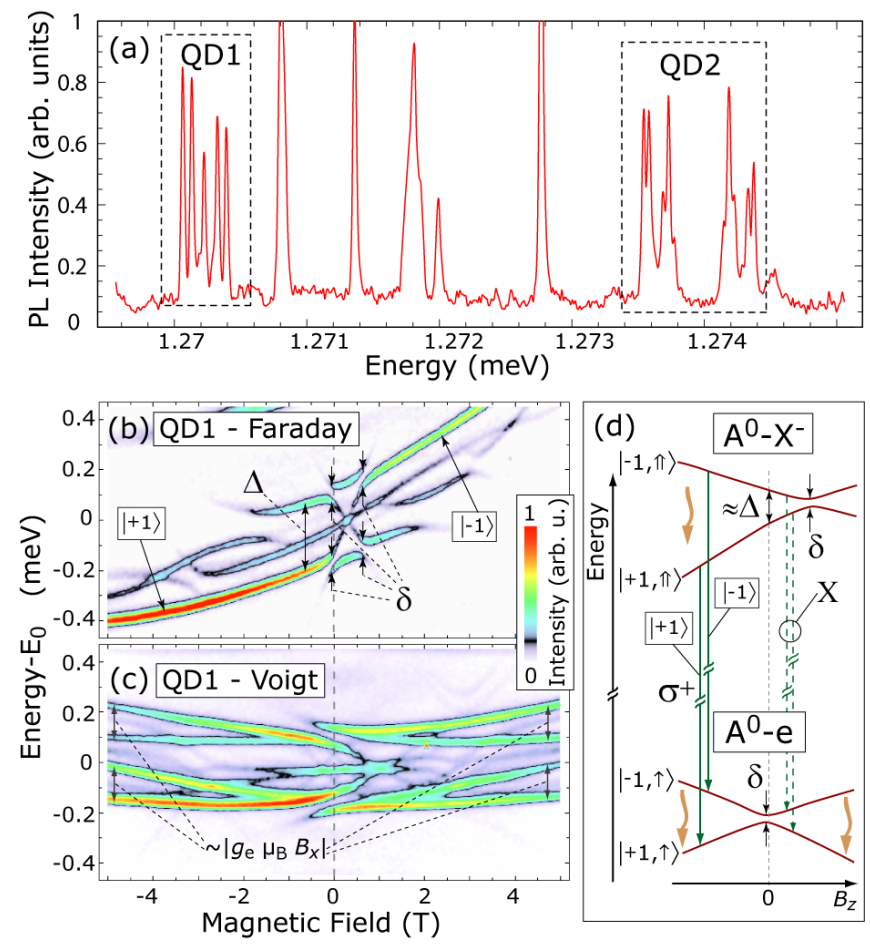

FIG. 1. (a) p-PL spectrum in zero magnetic field showing two sets of correlated lines (dashed line frames) originating from a 1Mn-doped (QD1) and a 2Mn-doped (QD2) quantum dot. (b),(c) Density plots of QD1 PL intensity measured in $\sigma^{+}$ polarization as a function of the magnetic field in Faraday and Voigt configuration respectively $\left(E_{0}=1.2702 \mathrm{eV}\right)$. (d) Sketch of the most significant energy levels which contribute for QD1 to the $\sigma^{+}$signal in Faraday configuration. The magnetic fields at which the anticrossings $\delta$ take place (positive in $A^{0}-X^{-}$ state, near zero in $A^{0}$-e state) determine the position of the characteristic X-pattern.

that negative trions $X^{-}$(2 electrons, 1 hole) are photocreated under illumination. Since both electrons in $X^{-}$ are paired in a singlet, their spin dependent properties vanish, which allows us to regard a negative trion as a single hole.

During an experimental run performed to measure a 1Mn-doped quantum dot (QD1 in figure 1) we observed another set of spatially and spectrally correlated lines (QD2), which could be ascribed from its magnetic dependence to a 2Mn-doped QD. First we use QD1 to recall the typical features of a $1 \mathrm{Mn}$-doped QD revealed by a magnetic field in Faraday geometry [13, 15, 23]. In Fig. 1(b), the PL spectra consist of two main lines (labeled $| \pm 1\rangle$ ) which are split by an energy $\Delta$ and experience each two anticrossings $\delta$ with two weaker lines. Altogether they form a characteristic X-pattern. The second noteworthy feature is the PL intensity transfer from $|+1\rangle$ to $|-1\rangle$ $(|-1\rangle$ to $|+1\rangle)$ in a strong positive (negative) field. Both these observations can be understood with the help of Fig. 1(d) which illustrates the field dependence of the $\mid \pm 1, \Uparrow$ or $\uparrow\rangle$ energy levels in the initial $A^{0}-X^{-}$and fi- nal $A^{0}$-electron (or $A^{0}$-e) states. The X-pattern results from the $\delta$ anticrossing of the $| \pm 1\rangle A^{0}$ states due to their anisotropic coupling [13, 22]. Due to the different strengths of the $A^{0}$-e and $A^{0}$-h exchange interactions, the $\delta$ anti-crossing between the $| \pm 1\rangle$ states occur at different magnetic fields for the $A^{0}-X^{-}$and $A^{0}$-e states. For $A^{0}$-e, it remains near zero field because of small exchange interaction (usually below $\delta$ ), while for $A^{0}-X^{-}$state, it is shifted to the positive magnetic field $B \approx \Delta /\left(2 g_{A^{0}} \mu_{\mathrm{B}}\right)$, where $g_{A^{0}}$ denotes the effective $g$-factor of $A^{0}$ spin $J=1$ and $\mu_{\mathrm{B}}$ is the Bohr magneton [13, 15]. Near these anticrossings, optical transitions with $A^{0}$ spin-flip are partially allowed (dashed lines in Fig. 1(d)) which produces the X-pattern. At higher magnetic fields, $A^{0}$ magnetization, i.e. the thermal relaxation between $\mid \pm 1$, $\Uparrow$ or $\uparrow\rangle$ Zeeman-split levels (wavy arrows in Fig. 1(d)), results in the pronounced change in intensity of the corresponding $| \pm 1\rangle$ lines in Fig. 1(b).

The magnetic dispersion of QD2 PL spectrum (Fig. 2(a)) reveals 4 bright lines exhibiting features quite similar to those of 1Mn-doped QDs (X-pattern and magnetization in high fields). However, the splittings denoted $\Delta_{1}$ and $\Delta_{2}$ appear twice, and so do the X-patterns $X_{i}(i=1,2)$ which connect each $\Delta_{i}$-split pair of lines with faint lines anticrossing the bright lines at $B_{i}=$ $\Delta_{i} /\left(2 g_{A_{i}^{0}} \mu_{\mathrm{B}}\right)$. This observation strongly differs from the $\mathrm{X}$-pattern duplicate already observed in 1Mn-doped QDs due either to electrostatic fluctuations of the environment or to different charge state [13]. The fact that there is two different $\mathrm{X}$-patterns and that the magnetization takes place within the 4 bright lines as a whole (i.e. only the highest (lowest) line survives in high positive (negative) field) strongly supports the interpretation that QD2 is doped with two distinct acceptors $A_{i}^{0}(i=1,2)$, each characterized by an exchange splitting $\Delta_{i}$ and an anticrossing energy $\delta_{i}$. Within this framework, the 4 bright lines can be associated to the 4 acceptor state configurations $\left|J_{z_{1}^{\prime}, A_{1}^{0}}= \pm 1, J_{z_{2}^{\prime}, A_{2}^{0}}= \pm 1\right\rangle$ as indicated in Fig. 2(a). The spin Hamiltonian model discussed below fully agrees with this interpretation.

To determine the proper local strain tensor experienced by each acceptor, we measured the effect of the transverse magnetic field $B_{x}$. As previously reported for 1Mn-doped QDs [15], the Zeeman-split lines exhibit a strong circular polarization up to high magnetic fields both for QD1 (Fig. 1(c)) and QD2 (Fig. 3(a)). This is evidenced by the asymmetry of the spectra with respect to $B_{x}$. This property results from the exchange interaction between $A^{0}$ and the QD hole which acts as a giant effective magnetic field in the $z^{\prime}$ direction (close to $z$ ) associated with the strain experienced by $A^{0}$, and thus inhibits the hole spin mixing by the transverse field [15]. Conversely, the electron Zeeman effect is much less perturbed by the small $A^{0}$-e exchange, which yields $(\uparrow \pm \downarrow) / \sqrt{2}$ spin states split by $\sim\left|g_{e} \mu_{\mathrm{B}} B_{x}\right|$, see e.g. Fig. 1(c). For QD2 (Fig. 3(a)), we again observe two X-patterns connect- 

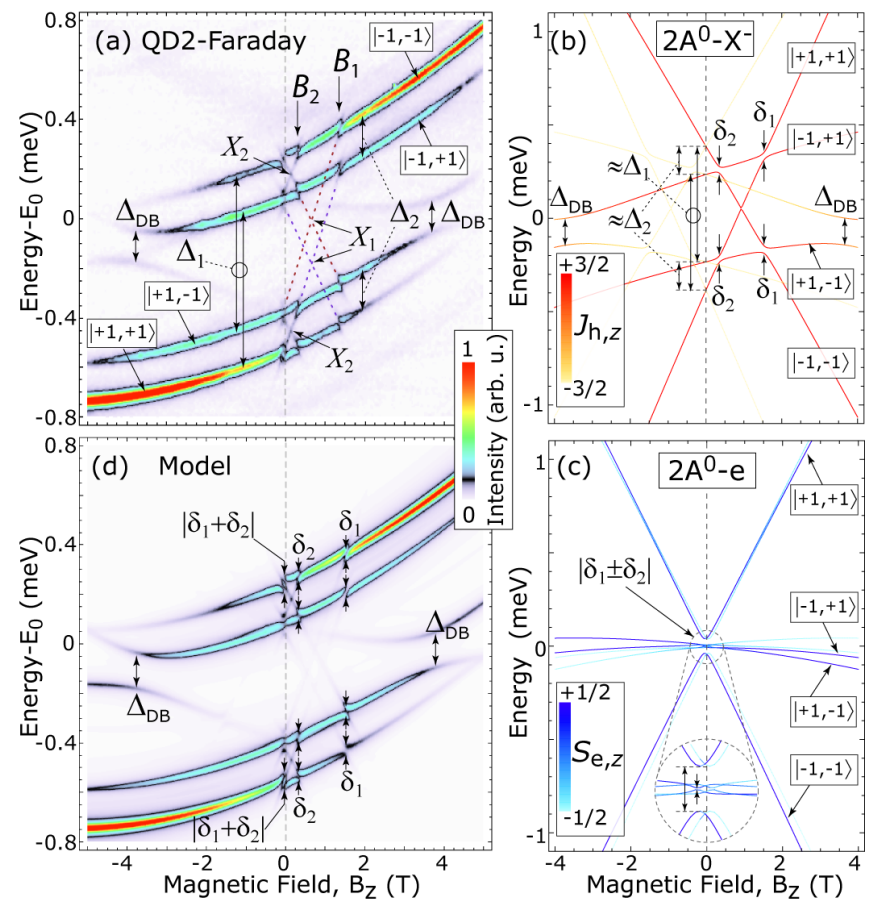

FIG. 2. (a) Density plot of QD2 PL intensity measured in $\sigma^{+}$polarization as a function of the magnetic field $B_{z}$ in Faraday configuration $\left(E_{0}=1.274 \mathrm{eV}\right)$. Dashed lines are drawn to make visible the $\mathrm{X}$-patterns $\left(X_{1}\right)$ associated to the anticrossings at $B_{1}$. (b),(c) Energy levels of $2 A^{0}-X^{-}$and $2 A^{0}$-e calculated as a function of $B_{z}$ and drawn with a color scale proportional to $J_{\mathrm{h}, z}$ and $S_{\mathrm{e}, z}$, respectively. (d) Theoretical density plot of the corresponding $X^{-}$PL intensity in $\sigma^{+}$polarization, assuming $T_{A^{0}}=11 \mathrm{~K}$.

ing the $\Delta_{2}$-split lines (thus analogous of $X_{2}$ in Faraday) which develop in negative fields. Much fainter lines crossing each other in the $-4 \mathrm{~T}$ field region (where they almost vanish) likely form two other $\mathrm{X}$-patterns connecting the $\Delta_{1}$-split lines (analogous of $X_{1}$ ). For QD1 (Fig. 1(d)), we observe in contrast a single X-pattern which moreover develops in positive fields. Since in Voigt geometry the $\mathrm{X}$-pattern position in the magnetic field is determined by the local strain tensor experienced by $A^{0}[15]$, one can conclude that both Mn-doped QDs do not share a common $A^{0}[24]$, even though they appear in the same $\mu-\mathrm{PL}$ spectra. Simulation of QD2 spectra can thus be achieved by assuming that QD2 contains two distinct $A^{0}$ in its own volume.

In previous works, we developed a general spin Hamiltonian model in which $A^{0}$ dopants were described as two interacting spins provided by the $3 d^{5}$ electrons $(S=5 / 2)$ and the Mn-bound hole $\left(J_{\mathrm{h}}=3 / 2\right)[13,15]$. Here, for simplicity, we treat each $A^{0}$ acceptor as an effective spin $J=1$. It reduces the Hilbert space dimension of the $A^{0}$ pair from $576(24 \times 24)$ to $9(3 \times 3)$, and turns out to be quite sufficient in moderate magnetic field to reproduce the acceptor levels in the observed $J=1$ configuration.
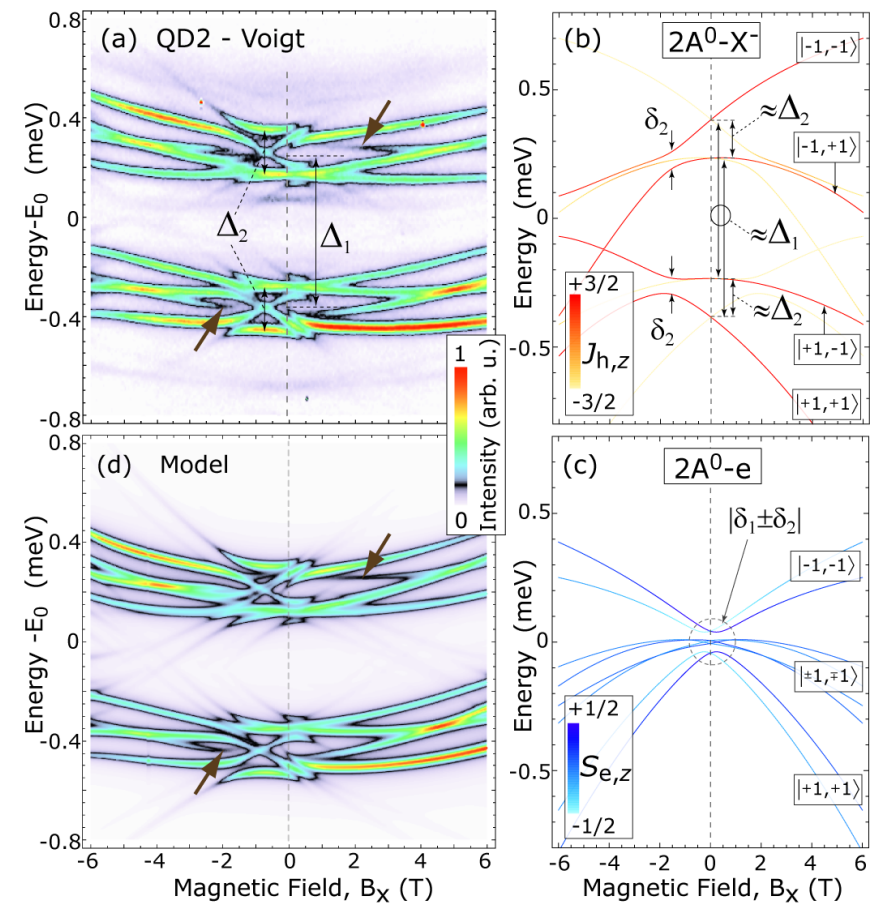

FIG. 3. (a) Density plot of QD2 PL intensity measured in $\sigma^{+}$polarization as a function of the magnetic field $B_{x}$ in Voigt configuration $\left(E_{0}=1.274 \mathrm{eV}\right)$. (b),(c) Energy levels of $2 A^{0}$-hole and $2 A^{0}$-electron calculated as a function of $B_{x}$ and drawn with a color scale proportional to $J_{\mathrm{h}, z}$ and $S_{\mathrm{e}, z}$, respectively. (d) Theoretical density plot of the corresponding $X^{-} \mathrm{PL}$ intensity in $\sigma^{+}$polarization, assuming $T_{A^{0}}=8 \mathrm{~K}$.

Our model still includes a strain-induced zero field splitting of the $J=1$ states specific to each $A_{i}^{0}(i=1,2)$, which formally reads $[15,25]$

$\hat{\mathcal{H}}_{\text {str }}=\sum_{i=1}^{2} \frac{-2 \Delta_{i}^{0}}{3}\left[\hat{J}_{z_{i}^{\prime}}^{2}-\frac{1}{2}\left(\hat{J}_{x_{i}^{\prime}}^{2}+\hat{J}_{y_{i}^{\prime}}^{2}\right)\right]+\frac{\delta_{i}}{2}\left(\hat{J}_{x_{i}^{\prime}}^{2}-\hat{J}_{y_{i}^{\prime}}^{2}\right)$.

The first term in Eq. 1 amounts to a tensile strain along the $z_{i}^{\prime}$ direction (defined by arbitrary polar angles $\theta_{s, i}$ and azimuthal angles $\left.\phi_{s, i}\right)$ blue-shifting by $\Delta_{i}^{0}$ the $|0\rangle$ level of $A_{i}^{0}$ from the $| \pm 1\rangle$ ones. The latter are further split by $\delta_{i}$ due to the second term of Eq. 1 which amounts to a shear strain in the plane perpendicular to $z_{i}^{\prime}$. For the QD-confined hole, a similar Hamiltonian (but without inplane shear strain) is used to reproduce the few $10 \mathrm{meV}$ $\Delta_{\mathrm{HL}}$ splitting between the heavy-hole $\left(J_{\mathrm{h}, z}= \pm 3 / 2\right)$ and light-hole $\left(J_{\mathrm{h}, z}= \pm 1 / 2\right)$ states [15]. The Zeeman effect is included for all individual spins : the hole and electron $g$-factors are deduced from the linear dispersion of the bright lines in Voigt and Faraday measurements, to $g_{\mathrm{h}}=0.65$ and $g_{\mathrm{e}}=-0.4$, respectively, while both $A^{0} g$-factors are fitted to reproduce the slopes of the faint lines in the X-patterns. Heisenberg-type exchange interactions, $\varepsilon_{\mathrm{h}, i} \hat{\boldsymbol{J}}_{i} \cdot \hat{\boldsymbol{J}}_{\mathrm{h}}$ or $\varepsilon_{\mathrm{e}, i} \hat{\boldsymbol{J}}_{i} \cdot \hat{\boldsymbol{S}}_{\mathrm{e}}(i=1,2)$, are assumed between both $A_{i}^{0}$ effective spins and the hole 
or electron spin, respectively. A specific anisotropic exchange $\hat{\mathcal{H}}_{\mathrm{a}, i}=\rho_{i} \varepsilon_{\mathrm{h}, i}\left(\hat{J}_{\mathrm{h}, z_{i}^{\prime \prime}}^{2} \hat{\boldsymbol{J}}_{i} \cdot \hat{\boldsymbol{J}}_{\mathrm{h}}+\hat{\boldsymbol{J}}_{i} \cdot \hat{\boldsymbol{J}}_{\mathrm{h}} \hat{J}_{\mathrm{h}, z_{i}^{\prime \prime}}^{2}\right)$ is also taken into account to include the hole valence band mixing at the $A_{i}^{0}$ sites [26]. This term which introduces the anisotropy strength $\rho_{i}$ and the direction $z_{i}^{\prime \prime}$ from the QD center to the $A_{i}^{0}$ site (defined by angles $\theta_{\mathrm{a}, i}$ and $\phi_{\mathrm{a}, i}$ ), is required only to reproduce the dark-bright splitting $\Delta_{\mathrm{DB}}$ observed around $\pm 3.5 \mathrm{~T}$ (Fig. 2(a)) $[13,15]$. The total spin Hamiltonians of the initial $\left(2 A^{0}-X^{-} \equiv 2 A^{0}\right.$-hole $)$ and final $\left(2 A^{0}\right.$-electron) states are each diagonalized for a series of magnetic fields as reported in Fig. 2(b)-(c) and Fig. 3(b)-(c). Note a specific color scale, which highlights the levels contributing to $\sigma^{+}$polarized recombination, is used to encode the hole spin $J_{h, z}$ and electron spin $S_{e, z}$. From the arrays of calculated eigenvalues and eigenvectors, we can generate the theoretical PL emission spectra simulating the experimental ones, by following the procedure described in Ref. 15 in order to include $A^{0}$ thermalization, diamagnetic shift and experimental broadening.

TABLE I. Parameters used for theoretical simulations. $\Delta_{i}^{0}$ in $\mathrm{meV}, \delta_{i}, \varepsilon_{\mathrm{h}, i}$ and $\varepsilon_{\mathrm{e}, i}$ in $\mu \mathrm{eV}, \theta_{s, i}, \phi_{s, i}, \theta_{a, i}$, and $\phi_{a, i}$ in degrees $\left({ }^{\circ}\right)$.

\begin{tabular}{l|ccccccccccc}
\hline \hline & $\Delta_{i}^{0}$ & $\delta_{i}$ & $\theta_{s, i}$ & $\phi_{s, i}$ & $g_{A_{i}^{0}, \|}$ & $g_{A_{i}^{0}, \perp}$ & $\varepsilon_{\mathrm{h}, i}$ & $\varepsilon_{\mathrm{e}, i}$ & $\rho_{i}$ & $\theta_{\mathrm{a}, i}$ & $\phi_{\mathrm{a}, i}$ \\
$A_{i=1}^{0}$ & 5 & 40 & 30 & 130 & 4 & 2.2 & -380 & 25 & -0.24 & 95 & -100 \\
$A_{i=2}^{0}$ & 3.5 & 35 & 25 & -120 & 4 & 3.2 & -55 & 15 & 0 & - & - \\
\hline \hline
\end{tabular}

The fitting parameters used in Fig. 2(d) and Fig. 3(d) are presented in Tab. I. Despite the simplicity of the acceptor description as a $J=1$ spin, this model reproduces accurately the experimental observations in both field configurations with a single set of parameters. Note however the $A^{0} g$-factors have to be taken explicitly anisotropic $\left(g_{A^{0}, \perp} \neq g_{A^{0}, \|}\right)$, whilst in a more elaborated model the sole strain-induced fine structure (Eq. 1) was enough to explain the magnetic anisotropy of $1 \mathrm{Mn}$-doped QDs [15]. Since the $A_{i}^{0}$-hole exchange is large compared to the $A_{i}^{0}$-electron exchange $\left(\right.$ i.e. $\left.3\left|\varepsilon_{\mathrm{h}, i}\right| \gg\left|\varepsilon_{\mathrm{e}, i}\right|\right)[13,16]$, as confirmed by the weakly perturbed electron Zeeman splitting in Voigt configuration, we can unequivocally determine the exchange parameters from the size and position in magnetic field of the X-patterns. Note that without the anisotropic term $\hat{\mathcal{H}}_{\mathrm{a}, i}$, we expect that $\left|\Delta_{i}\right| \approx$ $\left|3 \varepsilon_{\mathrm{h}, i}-\varepsilon_{\mathrm{e}, i}\right|$ which is verified for $A_{2}^{0}$ since $\rho_{2}=0$ [27]. With the help of Fig. 2(b),(c), one can now identify the four $2 A^{0}$ spin configurations giving rise to the four bright lines in the $\sigma^{+}$magneto-PL image : two ferromagnetic levels $| \pm 1, \pm 1\rangle$ which rapidly shift with the field, and two anti-ferromagnetic $| \pm 1, \mp 1\rangle$ levels with a weaker magnetic dispersion (due to the sole hole or electron Zeeman term). The origin of the four X-patterns becomes clear in Fig. 2(b) : each anticrossing $\delta_{i}$ experienced by the acceptor $A_{i}^{0}$ appears twice in the level diagram due to both possible orientations $| \pm 1\rangle$ of the second dopant $A_{j \neq i}^{0}$.
In Voigt geometry (Fig. 3(b),(c)), the magnetic dispersion is reduced due to the QD anisotropy, but is still dominated by the $2 A^{0}$ Zeeman effect associated with the finite projection of the external field $B_{x}$ onto the actual quantization axis $z_{i}^{\prime}$ of $A_{i}^{0}$ spin [15]. The hole keeps a pronounced $J_{\mathrm{h}, z}= \pm 3 / 2$ character up to high fields thanks to the large $2 A^{0}$-induced spin splittings $\approx\left|\Delta_{1} \pm \Delta_{2}\right|$ of a few $100 \mu \mathrm{eV}$ 's. Conversely, for the electron, the small $2 A^{0}$-electron exchange $\left(\varepsilon_{\mathrm{e}, i} \sim 20 \mu \mathrm{eV}\right)$ compares with the transverse Zeeman term only up to about $2 \mathrm{~T}$. In this low field region, the spectral details (see e.g. the marked lines in Fig. 3(a),(d)) provide sensitive criteria to finely adjust the corresponding $\varepsilon_{\mathrm{e}, i}$ parameters, in agreement with the estimate from the Faraday measurements.

A remarkable feature of such a $2 \mathrm{Mn}$-doped quantum dot is to provide a hole-mediated coupling between both $A^{0}$ spins. Indeed, at zero magnetic field (see Fig. 2(b) or 3(b)), the exchange interaction with the photo-created hole results in a ferromagnetic ground state $| \pm 1, \pm 1\rangle$. Thermalization of the $2 A^{0}$ spins in the presence of the hole induces thus a positive spin-spin correlation $\chi_{12}=$ $\left\langle\hat{\boldsymbol{J}}_{1} \cdot \hat{\boldsymbol{J}}_{2}\right\rangle$, as would be produced by a ferromagnetic exchange between the $A^{0}$ spins. By assuming a Boltzmann distribution, we calculated $\chi_{12}$ as a function of temperature for both the hole-mediated coupling, and a direct exchange $\varepsilon_{12} \hat{\boldsymbol{J}}_{1} \cdot \hat{\boldsymbol{J}}_{2}$ between $A^{0}$ spins (with $\varepsilon_{\mathrm{h}, 1}=\varepsilon_{\mathrm{h}, 2}=0$ ) [14]. A good agreement between the two correlation functions was obtained in the $0-10 \mathrm{~K}$ temperature range with $\varepsilon_{12} \simeq-70 \mu \mathrm{eV}$. For comparison, the actual direct exchange $\left|\varepsilon_{12}\right|$ is estimated in QD2 to be less than $5 \mu \mathrm{eV}$ [28]. Note in our measurements there is no obvious spin-spin correlation in the zero-field PL spectrum, likely because of the $X^{-}$lifetime and the $A^{0}$ effective temperature of about $10 \mathrm{~K}$. A more favorable regime to observe this correlation should be reached at a gate voltage where the quantum dot is charged by a resident hole.

In conclusion, we demonstrate the experimental coupling of two acceptor-type magnetic dopants by a photocreated hole in an InAs/GaAs quantum dot. Our study relies on the quantitative analysis of the magneto-PL images from a quantum dot doped with $2 \mathrm{Mn}$ atoms by using a simplified spin Hamiltonian model. It shows that both neutral magnetic dopants behave each like a 2-level systems giving rise altogether to $2 \times 2$ spin configurations. Combining this feature with the possibility to optically control a single spin [16] would open a way to achieve an optically-triggered spin-based CNOT gate. Besides, the hole-mediated coupling between the magnetic dopants also opens an original approach to optically investigate Zener-type ferromagnetism at the nanometer scale. 
[1] M. Atatüre, J. Dreiser, A. Badolato, A. Högele, K. Karrai, and A. Imamoglu, Science 312, 551 (2006).

[2] J. Berezovsky, M. H. Mikkelsen, N. G. Stoltz, L. A. Coldren, and D. D. Awschalom, Science 320, 349 (2008).

[3] B. D. Gerardot, D. Brunner, P. A. Dalgarno, P. Oehberg, S. Seidl, M. Kroner, K. Karrai, N. G. Stoltz, P. M. Petroff, and R. J. Warburton, Nature 451, 441 (2008).

[4] D. Press, T. D. Ladd, B. Zhang, and Y. Yamamoto, Nature 456, 218 (2008).

[5] E. D. Kim, K. Truex, X. Xu, B. Sun, D. G. Steel, A. S. Bracker, D. Gammon, and L. J. Sham, Phys. Rev. Lett. 104, 167401 (2010).

[6] T. M. Godden, J. H. Quilter, A. J. Ramsay, Y. Wu, P. Brereton, S. J. Boyle, I. J. Luxmoore, J. Puebla-Nunez, A. M. Fox, and M. S. Skolnick, Phys. Rev. Lett. 108, 017402 (2012).

[7] K. Müller, A. Bechtold, C. Ruppert, C. Hautmann, J. S. Wildmann, T. Kaldewey, M. Bichler, H. J. Krenner, G. Abstreiter, M. Betz, and J. J. Finley, Phys. Rev. B 85, 241306 (2012).

[8] H. J. Krenner, M. Sabathil, E. C. Clark, A. Kress, D. Schuh, M. Bichler, G. Abstreiter, and J. J. Finley, Phys. Rev. Lett. 94, 57402 (2005).

[9] A. N. Vamivakas, C.-Y. Lu, C. Matthiesen, Y. Zhao, S. Fält, A. Badolato, and M. Atatüre, Nature 467, 297 (2010).

[10] L. Robledo, L. Childress, H. Bernien, B. Hensen, P. F. A. Alkemade, and R. Hanson, Nature 477, 574 (2011).

[11] A. Greilich, S. G. Carter, D. Kim, A. S. Bracker, and D. Gammon, Nature Photonics 1, 1 (2011).

[12] L. Besombes, Y. Léger, L. Maingault, D. Ferrand, H. Mariette, and J. Cibert, Phys. Rev. Lett. 93, 207403 (2004).
[13] A. Kudelski, A. Lemaître, A. Miard, P. Voisin, T. C. M. Graham, R. J. Warburton, and O. Krebs, Phys. Rev. Lett. 99, 247209 (2007).

[14] L. Besombes, C. L. Cao, S. Jamet, H. Boukari, and J. Fernández-Rossier, Phys. Rev. B 86, 165306 (2012).

[15] O. Krebs, E. Benjamin, and A. Lemaître, Phys. Rev. B 80, 165315 (2009).

[16] E. Baudin, E. Benjamin, A. Lemaître, and O. Krebs, Phys. Rev. Lett. 107, 197402 (2011).

[17] F. Marczinowski, J. Wiebe, J.-M. Tang, M. E. Flatté, F. Meier, M. Morgenstern, and R. Wiesendanger, Phys. Rev. Lett. 99, 157202 (2007).

[18] D. Kitchen, A. Richardella, J.-M. Tang, M. E. Flatté, and A. Yazdani, Nature 442, 439 (2006).

[19] J. Schneider, U. Kaufmann, W. Wilkening, M. Baeumler, and F. Köhl, Phys. Rev. Lett. 59, 240 (1987).

[20] N. Averkiev, A. Gutkin, and E. Osipov, Soviet Physics Semiconductors 21, 1119 (1987).

[21] A. Bhattacharjee, Sol. Stat. Comm. 113, 17 (1999).

[22] A. O. Govorov, Phys. Rev. B 70, 35321 (2004).

[23] J. van Bree, P. M. Koenraad, and J. Fernández-Rossier, Phys. Rev. B 78, 165414 (2008).

[24] A common $A^{0}$ located between two close-lying QDs could possibly interact with the evanescent part of the hole wavefunctions in both QDs.

[25] A. M. Yakunin et al., Nat. Mat. 6, 512 (2007).

[26] A. K. Bhattacharjee, Phys. Rev. B 76, 75305 (2007).

[27] The $\Delta_{\text {DB }}$ splitting could be well reproduced with the $A_{1}^{0}$ anisotropic term only.

[28] When included in the model, the direct exchange $\left|\varepsilon_{12}\right|$ induces a relative magnetic shift of the two similar $X$ patterns $\left(X_{1}\right.$ or $\left.X_{2}\right)$ by $\varepsilon_{12} /\left(g_{A^{0},||} \mu_{\mathrm{B}}\right)$, which is not observed experimentally. 